[16] Osborne et Wakeman. Journ. of Biol. Ohem., 1915, 21, 539; et 1916, $28,1$.

[17] Röse-Gottlieb. Le Lait, 1926, 6, 54.

[18] Sóhlossmann. Ztschr. f. Physiolog. Chemie, 1905, 47, 327.

[19] Stoklasa (J.). Ztschr. f. Physiolog. Chemie, 1897, 23, 343.

[20] Tolmatscheff. Hoppe-Seyler's med. chem. Unters., 1867, 2, 272.

[21] Trautmann et Krrchнor. Biochem. Ztschr., 1932, 247, 275.

[22] Woods et KocH. Journ, of Biol. Chem., 1905-1906, 1, 203.

\title{
ÉTUDE MICROBIOLOGIQUE SUR LA FILTRATION DU LAIT
}

\author{
par Dr JAROSLAV MASEK
}

De l'Institut lactologique de l'Ecole Polytechnique à Prague.

Directeur : Professeur D\& Otakar LAxa (Tehécoslovaquie).

Il existe toute une série de travaux sur l'influence de la filtration sur la teneur en impuretés du lait, mais la composition bactériologique du lait n'a été que très rarement traitée. C'est pour cela que je me suis décidé à étudier, quels changements microbiologiques se produisent pendant la filtration du lait. J'ai recueilli des échantillons de lait aussitôt après la traite et j'ai analysé le lait avant et après filtration. Pour cette étude, je me suis servi chaque fois du lait d'une seule vache. J'ai déterminé le nombre de germes par la méthode des plaques avec gélose au petit-lait. En même temps, j'ai observé le nombre des bactéries de la fermentation lactique pour cette raison : ces germes forment très souvent des chaînettes et il est intéressant de savoir quel rôle jouent ees groupes de bactéries. pendant la filtration. Probablement ces plus importants groupes de bactéries sont retenus sur le filtre. J'ai employé le filtre d'ouate "Simplex" qui a été, chaque fois, après le travail, convenablement nettoyé avec une solution sodée chaude, rincé et couvert, pour être préservé contre toute infection. Les essais ont été faits dans la ferme " $\mathrm{A}$ " dans l'automne et l'hiver de l'année 1934.

Les résultats sont présentés dans le tableau I. Par centimètre cube de lait non filtré, j'ai trouvé de 835 à 54.650 , en moyenne 16.929 germes, tandis que dans le même lait filtré, 1.620 à 32.500 , en moyenne 8.751 germes. Ainsi, nous voyons que le nombre des germes diminue par la filtration, mais pas nécessairement ; dans les deux échantillons de lait filtré on a constaté la présence de plus nombreux germes après la filtration qu'avant. Pour avoir une détermination plus précise de ces changements, je calcule le degré microbiologique de la filtration : e'est le nombre de germes dans le lait filtré calculé en \% par rapport au nombre de germes dans le même lait non filtré ; c'est ce degré qui indique de combien est meilleure ou pire la com- 
position microbienne du lait après sa filtration. Ce degré atteint, dans les échantillons analysés, 33,50 - 194,01, en moyenne $85^{\circ} 39$. Dans le meilleur cas, le nombre des germes diminue d'un tiers ; mais, dans le plus mauvais, il augmente du double.

Le tableau I montre les changements survenus dans le nombre des germes qui produisent l'acide lactique. On en a trouvé dans le lait non filtré, de 65 à 17.500 , soit en moyenne 7.083 , c'est-à-dire $7,78-37,67$, en moyenne $25,38 \%$, par rapport à l'ensemble des

TABLEAU I

\section{LA FILTRATION TOUT DE SUITE APRÈS LA TRAITE}

\begin{tabular}{|c|c|c|c|c|c|c|c|}
\hline \multirow{3}{*}{ No } & \multicolumn{3}{|c|}{ Avant la filtration } & \multicolumn{3}{|c|}{ Après la filtration } & \multirow{3}{*}{$\begin{array}{l}\text { Le degré } \\
\text { miero- } \\
\text { bien de la } \\
\text { filtration }\end{array}$} \\
\hline & \multirow{2}{*}{$\begin{array}{c}\text { Tous les } \\
\text { germes } \\
\text { par centi- } \\
\text { mètre } \\
\text { cube }\end{array}$} & \multicolumn{2}{|c|}{$\begin{array}{l}\text { Les bactéxies } \\
\text { lactiques }\end{array}$} & \multirow{2}{*}{$\begin{array}{c}\text { Tous les } \\
\text { germes } \\
\text { par centi- } \\
\text { mètre } \\
\text { cube }\end{array}$} & \multicolumn{2}{|c|}{$\begin{array}{c}\text { Les bactóries } \\
\text { lactiques }\end{array}$} & \\
\hline & & $\begin{array}{c}\text { par centi- } \\
\text { mètre } \\
\text { cube }\end{array}$ & $\begin{array}{c}\% \text { de } \\
\text { tous les } \\
\text { germes }\end{array}$ & & $\begin{array}{l}\text { par cen- } \\
\text { timètre } \\
\text { cube }\end{array}$ & $\begin{array}{c}\% \text { de } \\
\text { tous les } \\
\text { germes }\end{array}$ & \\
\hline $1 \ldots \ldots \ldots$ & 22.200 & pas & noté & 9.497 & pas & noté & 42,78 \\
\hline $2 \ldots \ldots \ldots \ldots$ & 3.510 & & & 5.360 & & & 152,71 \\
\hline $3 \ldots \ldots \ldots$ & 10.805 & 4.070 & 37,67 & 6.000 & 2.060 & 34,33 & 55,53 \\
\hline 4 & 6.995 & 2.610 & 37,31 & 3.075 & 670 & 21,79 & 43,96 \\
\hline $5 \ldots \ldots$ & 54.650 & 14.600 & 26,71 & 32.500 & 7.750 & 23,85 & 59,47 \\
\hline $6 \ldots \ldots \ldots$ & 48.650 & 17.500 & 35,97 & 16.300 & 2.350 & 14,42 & 33,50 \\
\hline $7 \ldots \ldots \ldots$ & 2.685 & 335 & 12,47 & 2.530 & 280 & 11,07 & 94,23 \\
\hline 8 & 2.030 & 400 & 19,72 & 1.875 & 240 & 12,80 & 92,36 \\
\hline $9 \ldots \ldots \ldots$ & 835 & 65 & 7,78 & 1.620 & 170 & 10,49 & 194,01 \\
\hline Moyenne ...... & 16.929 & 7.083 & 25,38 & 8.751 & 1.932 & 18,39 & 85,39 \\
\hline Minimum .... & 835 & 65 & 7,78 & 1.620 & 170 & 10,49 & 33,50 \\
\hline Maximum ... & 54.650 & 17.500 & 37,67 & 32.500 & 7.750 & 34,33 & 194,01 \\
\hline & & & & & & & \\
\hline
\end{tabular}

germes, mais dans le même lait filtré seulement 170 ̀े 7.750 , en moyenne 1.932 germes, ou 10,49 à 34,33 , en moyenne $18,39 \%$, de la flore totale. Dans un seul cas, le nombre de ces germes a augmenté après la filtration, mais seulement de 105 ; au contraire, dans les autres échantillons, on peut voir la diminution survenant à la suite de la filtration du lait - le taux passant dans un échantillon, de 17.500 à 2.350 .

Le fait qu'après la filtration, nombre de bactéries lactiques manquaient, peut être expliqué ainsi : les chaînettes de ces bactéries ont été arrêtées par le filtre (ouate) ou, du moins en grande partie, et d'autant plus que le développement des chaînettes était plus grand. Voici pourquoi, peut-être, dans l'échantillon no 9, qui se dis- 
tinguait par un petit nombre de microorganismes produisant de l'acide lactique, la formation des chaînettes n'avait pas été suffisante et celles-ci ne pouvaient pas être arrêtées sur le filtre.

Dans le tableau I, on peut voir aussi que les échantillons nos 3-6 contenant, avant la filtration, beaucoup de germes de la fermentation lactique $(26,71-37,67 \%)$ se distinguent, après la filtration, par une remarquable diminution du nombre de tous les germes (le degré microbien comprenait $33^{\circ} 50-59^{\circ} 47$ ). Ces échantillons du lait, avec un nombre plus bas des bactéries de l'acide lactique (7,78-19,72\%), ne montrent plus l'influence si favorable de la filtration du lait (le degré microbien 'contenait $92^{\circ} 36-194^{\circ} 01$ ). Probablement, le nombre des bactéries de la fermentation lactique étant plus grand, le développement des chaînettes était plus important et leur arrêt sur l'ouate plus sensible.

Cette observation est complétée par l'examen de l'ouate déjà employée. L'ouate coupée en petits morceaux a été plongée dans une quantité fixe de solution physiologique stérilisée, celle-ci étant agitée pendant 10 minutes. De cette manière, les germes retenus sur l'onate ont été dispersés dans la solution. On a ensemencé, à partir de celle-ci, des plaques d'agar, et le nombre des germes a été calculé par rapport à la quantité de solution. Tandis que l'ouate non utilisée contenait seulement de 6 à 260 germes, c'est-à-dire un germe par centimètre carré (tableau II), on a trouvé sur l'ouate ayant servi de filtre pour le lait $2.180 .000,9.300 .000$ et 38.400 .000 germes. L'ouate pure ne contenait que des moisissures (Mucor et Penicillium),

TABLEAU II

LE FILTRE (OUATE) AVANT LA FLLTRATION

\begin{tabular}{|c|c|c|}
\hline No & Tous les germes du filtre & $\begin{array}{l}\text { Les germes sur un centimètre } \\
\text { carré de filtre }\end{array}$ \\
\hline $1 \ldots \ldots \ldots \ldots$ & 73 & $0-1$ \\
\hline $2 \ldots \ldots \ldots \ldots \ldots$ & 6 & $0-1$ \\
\hline $3, \ldots \ldots \ldots \ldots \ldots \ldots$ & 186 & $1-2$ \\
\hline $4 \ldots \ldots \ldots \ldots \ldots$ & 260 & $1-2$ \\
\hline
\end{tabular}

mais on a trouvé sur l'ouate déjà employée $1.000 .000,3.200 .000$ et 2.000 .000 de bactéries lactiques. Bien que nous sachions que tous les germes de l'ouate n'ont pas été dispersés dans la solution, les observations confirment l'idée que des bactéries lactiques ont été arrêtées sur le filtre d'ouate.

Assurément il faut étudier aussi l'infection du lait pendant la filtration. Nous supposons, qu'il y a sur le filtre beaucoup de germes malgré le nettoyage sérieux et ces germes peuvent être entraînés 
TABLEAU III

LE FILTRE (OUATE) APRÈS LA FILTRATION

\begin{tabular}{|c|c|c|c|}
\hline \multirow[b]{2}{*}{ No } & \multirow[b]{2}{*}{ Tous les germes } & \multicolumn{2}{|c|}{ Les bactéries lactiques } \\
\hline & & sur tout le filtre & $\begin{array}{c}\% \text { de tous les } \\
\text { germes }\end{array}$ \\
\hline 1 . & 2.180 .000 & 1.000 .000 & 45,87 \\
\hline 2 & 9.300 .000 & 3.200 .000 & 34,41 \\
\hline$\ldots$. & 38.400 .000 & 2.000 .000 & 5,21 \\
\hline
\end{tabular}

avec le lait, et ainsi le nombre des germes dans le lait filtré augmente. Pour savoir si cette infection est capable de changer la composition mierobienne du lait, ou si elle est un phénomène absolument insignifiant, j'ai rincé le filtre avec la solution physiologique stérilisée et puis j'ai déterminé dans celle-ci le nombre des germes retirés du filtre. L'essai a été répété (voir tableau IV). Sur l'appareil on a trouvé 286.500 et 435.000 germes. Si on filtrait seulement 10 litres de lait, le danger d'infection ne serait représenté que par 29 et 43 germes par eentimètre cube de lait. Le même essai a été répété après le nettoyage moins sérieux de l'appareil et en voici le résultat : sur le filtre, on a trouvé 1.590 .000 germes. Dans ces conditions, le danger d'infection par centimètre cube de lait serait représenté par 159 germes. Malgré cela, pratiquement, il faut dire que l'infection pendant la filtration pourrait, même dans cet exemple, influencer à peine les résultats de la composition microbienne constatée du lait. Il n'y a pas de germes de la fermentation lactique sur l'appareil, mais seulement des microorganismes de l'eau et de l'air, qui ne trouvent pas toujours dans le lait les conditions favorables à leur développement.

TABLEAU IV

L'INFEGTION PENDANT LA FILTRATION

\begin{tabular}{|c|c|c|}
\hline$=-$ & Tous les germes de l'appareil & $\begin{array}{l}\text { Degré d'infection pour } \\
\text { un centimètre cube de lait }\end{array}$ \\
\hline $1 \quad \ldots \ldots \ldots \ldots$ & 286.500 & 29 \\
\hline $2 \ldots \ldots \ldots \ldots \ldots \ldots$ & 435.000 & 43 \\
\hline $3 \ldots \ldots \ldots \ldots \ldots$ & 1.590 .000 & 159 \\
\hline
\end{tabular}

Les essais de la filtration du lait ne seraient pas complets, si on ne les comparait avec ceux provenant de circonstances qui se présentent pendant la purification du lait par la méthode le plus souvent employée : le "coulage" sur toile. Les résultats de ces essais sont dans le tableau V. Dans le lait original on a trouvé 58.500 et 
23.700, et dans le même lait filtré 85.500 et 77.600 germes. Voici une grande augmentation, le degré microbien du "coulage " fait 147086 et $327^{\circ} 43$. On a constaté dans le lait original 9.000 et 4.200 germes lactiques, soit 15,38 et $17,72 \%$, de la flore totale, dans le lait purifié,

TABLEAU $V$

LE COULAGE DU LAIT

\begin{tabular}{|c|c|c|c|c|c|c|c|}
\hline \multirow{3}{*}{ No } & \multicolumn{3}{|c|}{ Avant le coulage } & \multicolumn{3}{|c|}{ Après le coulage } & \multirow{3}{*}{$\begin{array}{l}\text { Le degré } \\
\text { micro- } \\
\text { bien }\end{array}$} \\
\hline & \multirow{2}{*}{$\begin{array}{c}\text { Tous les } \\
\text { germes } \\
\text { par cen- } \\
\text { timètre } \\
\text { cube }\end{array}$} & \multicolumn{2}{|c|}{ Les bactéries lactique: } & \multirow{2}{*}{$\begin{array}{l}\text { Tous les } \\
\text { germes } \\
\text { par cen- } \\
\text { timètre } \\
\text { cube }\end{array}$} & \multicolumn{2}{|c|}{ Les bactéries lactiques } & \\
\hline & & $\begin{array}{c}\text { par cen- } \\
\text { timètre } \\
\text { cube }\end{array}$ & $\begin{array}{c}\% \text { de } \\
\text { tous les } \\
\text { germes }\end{array}$ & & $\begin{array}{l}\text { par cen- } \\
\text { timètre } \\
\text { cube }\end{array}$ & $\begin{array}{c}\% \text { de } \\
\text { tous les } \\
\text { germes }\end{array}$ & \\
\hline $1 \ldots \ldots \ldots \ldots$ & 58.500 & 9.000 & 15,38 & 85.500 & 28.600 & 33,45 & 147,86 \\
\hline $2 \ldots \ldots \ldots \ldots$ & 23.700 & 4.200 & 17,72 & 77.600 & 18.000 & 23,20 & 327,43 \\
\hline
\end{tabular}

28.600 et 18.000 germes de la fermentation lactique ou 33,45 à $23,20 \%$. On ne peut pas remarquer l'arrêt des chaînettes microbiennes comme avec le filtre d'ouate, car il n'est pas assez grand et ne saurait être observé. Au contraire, l'augmentation du nombre des germes dans ces cas indique la dissociation des chaînettes, cette dissociation pouvant se produire aussi pendant la filtration, mais ne pouvant pas alors être remarquée, puisque l'ouate retient beaucoup de chaînettes.

Puis on a étudié cette question : les modifications de la flore microbienne (quant au nombre des germes et à la teneur en bactéries lactiques), qui accompagnent la filtration du lait, ont-ils ou non une influence au point de vue de la conservation du lait ? On a constaté que le lait non purifié coagule le lendemain, le lait filtré ou coulé le troisième jour ; les échantillons ayant été déposés à la température de la chambre. Outre cela, on a déposé quelques échantillons, après détermination du nombre des germes, à la température de la chambre et on a répété cette analyse toutes les 6 heures. Les résultats sont dans le tableau VI. On a trouvé par centimètre cube de lait non purifié, aussitôt après la traite, 835 à 22.200 germes, en moyenne 7.245 , après 6 heures, 2.285 à 146.000 , en moyenne 32.323 germes. Mais une grande augmentation des germes ne s'est produite que dans un seul cas (de 22.000 à 146.000); dans les autres échantillons, elle était faible, et, dans l'échantillon no 2 , nous trouvons même moins de germes le soir que dans le même lait de midi. Ici apparaît la phase bactéricide du lait, pendant laquelle la multiplication des germes est diminuée par l'action des agglutinines. Cette phase bactéricide 
TABLEAU VI

PHASE BACTÉRICIDE ET FLORE TOTALE

\begin{tabular}{|c|c|c|c|c|}
\hline \multirow{2}{*}{ No } & \multicolumn{2}{|c|}{ Non filtré } & \multicolumn{2}{|c|}{ Filtré } \\
\hline & Tout de suite & Après 6 heures & Tout de suite & Après 6 heures \\
\hline $1 \ldots \ldots \ldots \ldots$ & 22.200 & 146.000 & 9.497 & 126.000 \\
\hline $2 \ldots \ldots \ldots \ldots$ & 3.510 & 2.500 & 5.360 & 2.000 \\
\hline $3 \ldots \ldots \ldots \ldots$ & 6.995 & 8.100 & 3.075 & 1.300 \\
\hline $4 \ldots \ldots \ldots \ldots$ & 2.685 & 2.730 & 2.530 & 795 \\
\hline $5 \ldots \ldots \ldots \ldots$ & 835 & 2.285 & 1.620 & 6.770 \\
\hline Moyenne... & 7.245 & 32.323 & 4.416 & 27.373 \\
\hline
\end{tabular}

des divers laits dure plus ou moins longtemps, par exemple dans l'échantillon $n^{\circ} 1$, elle fut bientôt finie et l'augmentation des germes n'est pas troublée, au contraire dans les échantillons nos $2-4$ cette phase s'est prolongée jusqu'à la $6^{\mathrm{e}}$ heure après la traite. Etudions maintenant les mêmes effets dans le lait filtré. Tout de suite après la traite le lait contenait 1.620-9.497, en moyenne 4.416 germes. Puis, on trouve dans le lait filtré moins de germes que dans le lait non purifié, même après un certain temps. Dans les échantillons $n^{\text {os }} 2-4$ où la phase bactéricide a duré jusqu'après 6 heures, comme on l'a dit déjà, nous trouvons dans le lait filtré, après 6 heures, beaucoup moins de germes que tout de suite après la traite et la filtration; ce changement est beaucoup plus grand dans le lait filtré que dans le lait non purifié, où l'échantillon $n^{0} 2$ seul eontenait moins de germes après 6 heures qu'auparavant. C'est-à-dire que la phase bactéricide du lait filtré a été reconnue plus efficace que celle du lait non filtré. Il est intéressant de savoir s'il y a quelque relation entre la phase bactéricide et le nombre de bactéries lactiques. Voici pourquoi j'ai déterminé dans 4 échantillons le nombre de ces bactéries, les résultats figurent au tableau VII. On a trouvé dans le lait non

TABLEAU VII

LA PHASE BAGTERICIDE ET LES BAGTÉRIES DE LA FERMENTATION LAGTIQUE

\begin{tabular}{|c|c|c|c|c|}
\hline \multirow{2}{*}{$\mathrm{N}^{\circ}$} & \multicolumn{2}{|c|}{ Non filtré } & \multicolumn{2}{|c|}{ Filtré } \\
\hline & Tout de suite & Après 6 heures & Tout de suite & Après 6 heures \\
\hline $1 \ldots$ & 2.610 & 4.900 & 670 & 700 \\
\hline $2 \ldots \ldots \ldots \ldots$ & 335 & 365 & 280 & 75 \\
\hline $3 \ldots \ldots \ldots \ldots$ & 400 & 600 & 240 & 400 \\
\hline $4 \ldots \ldots \ldots \ldots \ldots$ & 65 & 100 & 170 & 100 \\
\hline Moyenne ..... & 852 & 1.491 & 340 & 319 \\
\hline
\end{tabular}


purifié, aussitôt après la traite, 65 à 2.610, en moyenne 852 , après 6 heures 100-4.900, en moyenne 1.491 bactéries lactiques. Leur multiplication est alors beaucoup moins ralentie que celle des autres germes (tableau VI). Quant au lait filtré, on a constaté, très peu de temps après la traite, 170-670, en moyenne 340,6 heures après 75-700, en moyenne 319 bactéries lactiques. Si on suppose que pendant la filtration du lait beaucoup de chaînettes microbiennes sont retenues, alors que généralement les bactéries dispersées ont passé dans le lait filtré, on arrive à la conclusion que les bactéries dispersées qui sont restées dans le lait fiłtré ont succombé surtout durant la phase bactéricide causée par les agglutines qui groupent les microbes, et c'est pour cette raison que le nombre de la flore totale a diminué. Et comme la phase bactéricide, quant aux bactéries lactiques, est pour le lait filtré d'un tel effet, la puissance conservative du lait filtré est beaucoup plus grande que celle du lait non filtré. Enfin, il reste à mentionner si la filtration du lait influe sur la quantité de Bacterium Coli commune dans le lait. J'ai déterminé ces germes dans les tubes de verre courbés, dans une solution de bile lactosée, avec l'addition d'un colorant sensible. Leur présence s'est manifestée par la formation de gaz et par la décoloration de la solution. Les résultats sont mentionnés dans le tableau VIII. On ne peut pas dire que la filtration a toujours une bonne influence. Dans les deux échantillons ( $\mathrm{n}^{\text {os }} 1-2$ ), on a trouvé certes une amélioration, mais dans d'autre cas une aggravation; quelquefois il n'y a aucun changement.

TABLEAU VIII

" BACTERIUM COLI COMMUNE "

(Est indiquée chaque fois, la dilution la plus grande du lait où il $\mathrm{y}$ a Bacterium coli commune.)

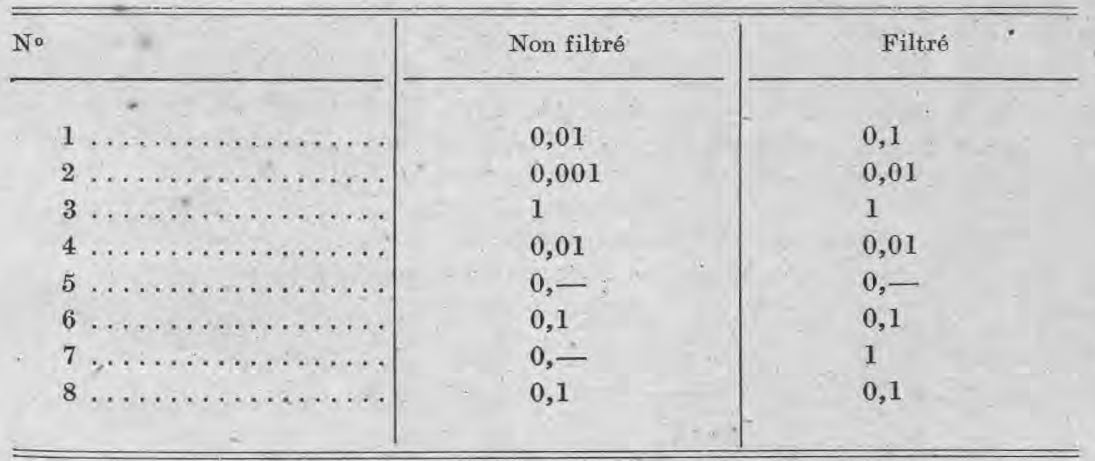

